\title{
Regulation of WWP2 Ubiquitin Ligase
}

Sandra B. Gabelli ${ }^{1}$, Zan Chen ${ }^{2}$, Hanjie Jiang ${ }^{2}$, Daniel Dempsey ${ }^{2}$, L. Mario Amzel ${ }^{3}$, Cynthia Wolberger ${ }^{3}$, Peter Devreotes ${ }^{4}$, Phil Cole $^{2}$ ${ }^{1}$ Medicine, Johns Hopkins University, Baltimore, United States, ${ }^{2}$ Pharmacology, Johns Hopkins University, Baltimore, United States, ${ }^{3}$ Biophysics and Biophysical Chemistry, Johns Hopkins University, Baltimore, United Kingdom, ${ }^{4}$ Cell Biology, Johns Hopkins University, Baltimore, United States E-mail: gabelli@jhmi.edu

The HECT E3 ligases ubiquitinate numerous transcription factors and signaling molecules and their activity must be tightly controlled to prevent cancer, immune disorders, and other diseases. In this study we have found that peptide linkers tethering WW domains in several HECT family members are key regulatory elements of their catalytic activities. Biochemical, structural, and cellular analysis has revealed that the linkers can lock the HECT domain in an inactive conformation and block the proposed allosteric ubiquitin binding site. Such linker-mediated autoinhibition of the HECT domain can be relieved by linker post-translational modifications, but complete removal of the brake can induce hyperactive autoubiquitination and E3 self-destruction. These results clarify the mechanisms of several HECT protein cancer associated mutations and provide a new framework for understanding how HECT ubiquitin ligases must be finely tuned to ensure normal cellular behavior.

Keywords: WWP2, ubiquitination, PTEN 